\title{
Neurogenetic Variations in Norepinephrine Availability Enhance Perceptual Vividness
}

\author{
Rebecca M. Todd, ${ }^{1}$ Mana R. Ehlers, ${ }^{1}$ Daniel J. Müller, ${ }^{2}$ Amanda Robertson, ${ }^{3}$-Daniela J. Palombo, ${ }^{3,4}$ Natalie Freeman, ${ }^{2}$ \\ Brian Levine, ${ }^{3,4}$ and Adam K. Anderson ${ }^{5}$ \\ ${ }^{1}$ Department of Psychology, University of British Columbia, Vancouver, British Columbia, V6T 1Z4, Canada, ${ }^{2}$ Department of Psychiatry, University of \\ Toronto and Neurogenetics Section, Centre for Addiction and Mental Health, Toronto, Ontario, M5T 1R8, Canada, ${ }^{3}$ Department of Psychology, University \\ of Toronto, Toronto, Ontario, M5S 3G3, Canada, ${ }^{4}$ Rotman Research Institute, Baycrest Health Sciences, Toronto, Ontario, M6A 2E1, Canada, and \\ ${ }^{5}$ Department of Human Development, Cornell University, Ithaca, New York 1485
}

Emotionally salient aspects of the world are experienced with greater perceptual vividness than mundane ones; however, such emotionally enhanced vividness (EEV) may be experienced to different degrees for different people. We examined whether BOLD activity associated with a deletion variant of the $A D R A 2 b$ gene coding for the $\alpha 2 \mathrm{~b}$ adrenoceptor modulates EEV in humans. Relative to noncarriers, $A D R A 2 b$ deletion carriers showed higher levels of perceptual vividness, with the ventromedial prefrontal cortex (VMPFC) showing greater modulation by EEV. Deletion carriers were also more sensitive to the featural salience of the images, suggesting a more pervasive role of norepinephrine in perceptual encoding. Path analysis revealed that, whereas a simple model by which the amygdala modulated the lateral occipital complex best characterized EEV-related activity in noncarriers, contributions of an additional VMPFC pathway best characterized deletion carriers. Thus, common norepinephrine-related neurogenetic differences enhance the subjective vividness of perceptual experience and its emotional enhancement.

Key words: ADRA2b; attention; emotion; emotionally enhanced vividness; fMRI; neurogenetics

\section{Introduction}

Emotionally salient stimuli typically evoke enhanced attention and memory compared with more mundane ones (Pourtois et al., 2013). Yet such emotional enhancement of cognition may be characterized by individual differences that are influenced by genotype (Hamann and Canli, 2004). A deletion variant of the $A D R A 2 b$ gene, which codes for the $\alpha 2 \mathrm{~b}$ adrenoceptor, is thought to be linked to similar effects to those of an $\alpha 2 \mathrm{~b}$ receptor antagonist (de Quervain et al., 2007). ADRA2b has been found to influence emotional enhancement of perception and memory (de Quervain et al., 2007; Rasch et al., 2009; Todd et al., 2013).

When salient stimuli are encountered, norepinephrine (NE) release from the locus ceruleus (LC) is associated with altered perceptual cortical activity (Jones and Moore, 1977; Aston-Jones and Cohen, 2005; Yu and Dayan, 2005; Donner and Nieuwenhuis, 2013). One potential consequence is altered salience of per-

Received Oct. 29, 2014; revised March 13, 2015; accepted March 16, 2015.

Author contributions: R.M.T., D.J.M., D.J.P., B.L., and A.K.A. designed research; R.M.T., M.R.E., and A.R. performed research; R.M.T., M.R.E., D.J.M., and N.F. analyzed data; R.M.T., M.R.E., and A.K.A. wrote the paper.

This research was supported by Canadian Institutes for Health Research (CIHR) Operating Grant 491746. D.J.M. is the recipient of a CIHR Michael Smith New Investigator Salary Prize and an OMHF New Investigator Fellowship. We thank Matthew Dixon for his generous and insightful comments and Sajid Shaikh, David Irwin, and Tayler Eaton for their contributions to the research.

The authors declare no competing financial interests.

Correspondence should be addressed to Rebecca Todd, PhD, Department of Psychology, University of British Columbia, 2136 West Mall, Vancouver, BC, V6T 124, Canada. E-mail: becket.todd@psych.ubc.ca.

D.J. Palombo's present address: VA Boston Healthcare System, 150 South Huntington Avenue, Boston, MA 02130. D0I:10.1523/JNEUROSCI.4489-14.2015

Copyright $@ 2015$ the authors $\quad 0270-6474 / 15 / 356506-11 \$ 15.00 / 0$ ceptual experience. We recently reported that emotionally salient stimuli are subjectively experienced with greater perceptual vividness (Todd et al., 2012), a phenomenon we call emotionally enhanced vividness (EEV). EEV has been linked to greater activation of object-sensitive regions of visual cortex, an effect mediated by amygdala activity (Todd et al., 2012). However, it is not known whether such interactions are related to NE availability.

The goal of the present study was to examine whether $A D R A 2 b$ genotype influences neural activity linked to EEV. fMRI data were collected from previously genotyped participants (Todd et al., 2013) while they performed a magnitude estimation task in which they estimated the relative amount of Gaussian noise overlaid on emotionally salient and neutral images (Fig. 1; Todd et al., 2012). Higher levels of subjective perceptual vividness are reflected in ratings of lower relative levels of noise. EEV is observed when emotionally salient images are rated as more perceptually vivid than neutral images.

The biased attention by NE (BANE) model (Markovic et al., 2014) stresses the role of NE in affective biases in attention, emphasizing reciprocal interactions between brain regions that play a key role in valuation networks - specifically the amygdala and ventromedial prefrontal cortices (VMPFC; Markovic et al., 2014; Fig. 2). According to this model, the LC/NE can modulate visual cortex activity both directly and via the amygdala and VMPFC (for review, see Andrews-Hanna et al., 2010; Pessoa, 2010; Chikazoe et al., 2014; Markovic et al., 2014). Thus, differences in NE activity may lead to greater activation of valuation network nodes and a greater influence of these re- 


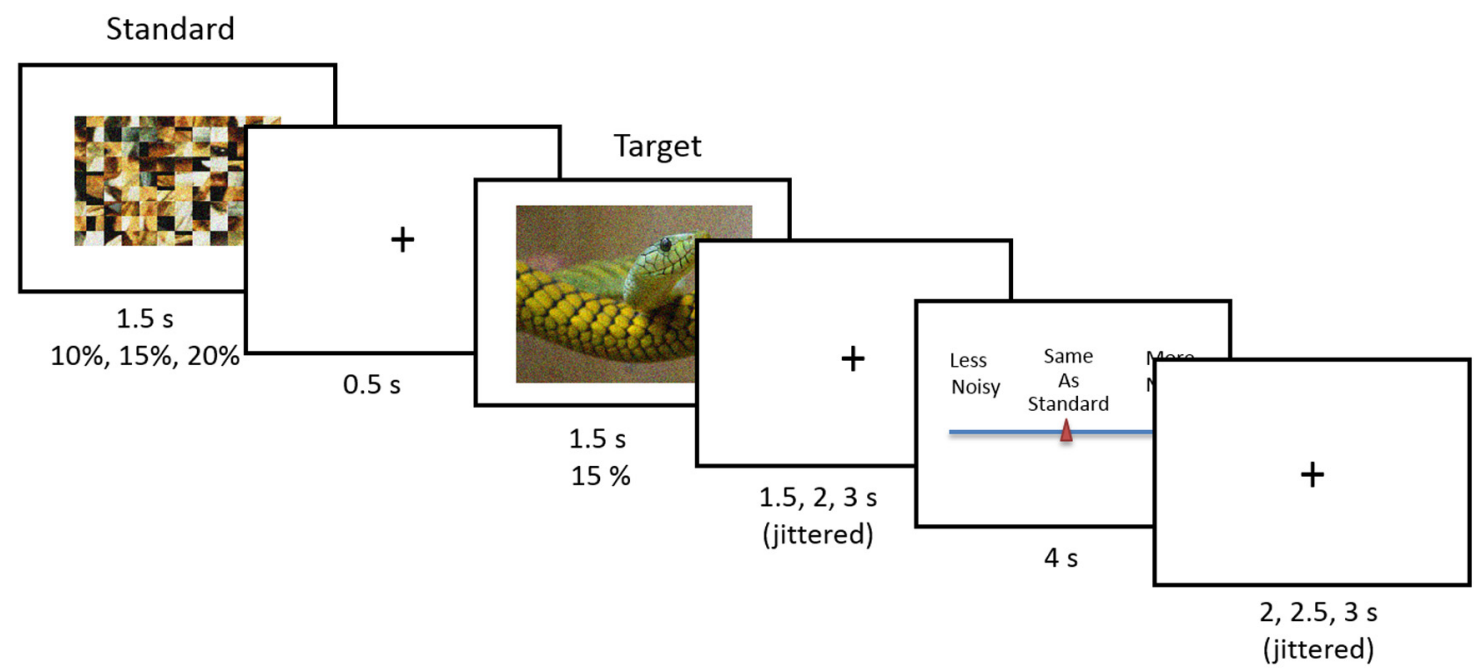

\section{2 seconds}

Figure 1. Task design for Noise Estimation fMRI experiment. A standard, created by phase scrambling the comparison image, was overlaid with 10\%, $15 \%$, or $20 \%$ noise. The standard was followed by the target image overlaid with $15 \%$ noise. Following image offset, participants moved a cursor on a scale to indicate noise for the image relative to the standard from "a lot less noise" to "same as standard" to "a lot more noise."

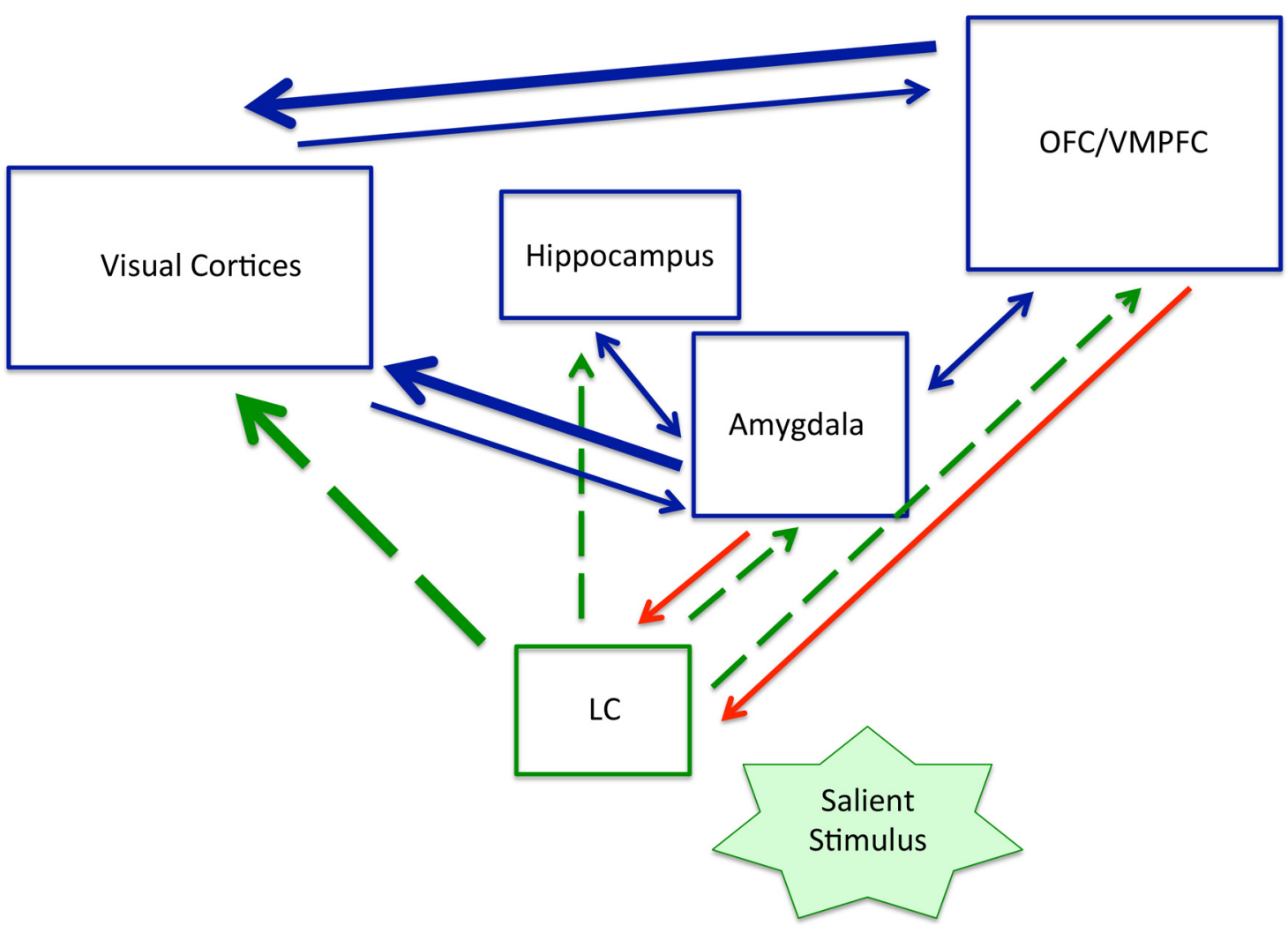

Figure 2. Key pathways emphasized by the BANE model (Markovic et al., 2014). Green dashed lines indicate norepinephrine (NE) pathways. Red lines indicate projections to the locus coeruleus (LC). Thicker lines indicate direct modulation of visual cortex activity in affect-biased attention. Norepinephrine (NE) activity is implicated in both stimulus encoding and selective attention (Sara, 2009). A salient stimulus activates LC neurons, which project widely to cortical and subcortical regions. OFC/VMPFC, Orbitofrontal/ventromedial prefrontal cortex. Reprinted from Behavioral and Brain Research. Copyright (2014), with permission from Elsevier.

gions on visual cortex activity. By combining genotyping with fMRI, we aimed to assess differences in EEV-related bloodoxygenation-level-dependent (BOLD) activation patterns linked to common differences in NE activity. We predicted that
$A D R A 2 b$ deletion carriers would show higher levels of EEV than noncarriers. We further predicted that ADRA2b-related differences in $\mathrm{EEV}$ would be associated with greater activation in the amygdala/VMPFC and a stronger pattern of coactiva- 
Table 1. Distribution of COMT and $5 H T T L P R$ genotypes among ADRA2 $b$ deletion carriers and noncarriers

\begin{tabular}{llllll}
\hline ADRA2b & COMTVal & COMT No Val & 5HTTLPR Short & 5HTTLPR Long & Total \\
\hline Del & 14 & 7 & 10 & 11 & 21 \\
No Del & 13 & 5 & 12 & 6 & 18 \\
Total & 27 & 12 & 22 & 17 & 39 \\
\hline
\end{tabular}

Del, deletion carriers; No Del, deletion noncarriers.

tion between these regions and visual cortex activity linked to EEV.

\section{Materials and Methods}

\section{Participants}

fMRI data were collected from 39 healthy, Caucasian young adult human participants (age: 18-35 years, 25 female) with normal or corrected-tonormal vision. These participants had been previously genotyped as part of a related study based at University of Toronto. Participants were excluded if they reported a history of psychiatric disorders, depression, and anxiety. Psychological health was confirmed by scores on the six item Kessler Psychological Distress scale (Kessler et al., 2002). All participants scores fell above the cutoff score of 14 (Cornelius et al., 2013). Participants were selected for equal numbers of each $A D R A 2 b$ genotype and grouped into $A D R A 2 b$ deletion carriers (homozygous and heterozygous, $N=21)$ and noncarriers $(N=18)$ who were matched for sex and working memory performance as measured by a visuospatial working memory task (Todd et al., 2014).

Variations in two other genes have also been associated with individual differences in affective biases. Carrying a short allele of the 5HTTLPR region of the serotonin transporter gene has been associated with trait neuroticism (Canli, 2008) and attentional biases and enhanced amygdala activation for threatening stimuli (Hariri and Weinberger, 2003; Munafò et al., 2008). A val158met polymorphism in the COMT gene influencing prefrontal dopamine metabolism is also associated with greater amygdala activation (Smolka et al., 2005) and startle responses to aversive stimuli (Montag et al., 2008). Given a high degree of reciprocal activity between NE, dopaminergic, and serotonergic systems (Sara and Bouret, 2012), we also examined these polymorphisms to control for their influence on EEV and associated fMRI activation. This allowed us greater ability to identify the extent to which observed effects are uniquely due to the influence of NE, and allowed us to conduct exploratory analyses probing effects of these genes on EEV. To the extent possible, $A D R A 2 b$ deletion carriers and noncarriers were matched for whether they carried the COMT met and 5HTTLPR short alleles. However, given the limitations of our genotyped participant pool, there were some inequalities in the distribution of 5HTTLPR genotype between $A D R A 2 b$ groups: among participants who did not carry the ADRA2b deletion variant there were substantially fewer 5HTTLPR long allele carriers than short allele carriers. In contrast, among $A D R A 2 b$ deletion carriers there were equal numbers of participants with and without the 5HTTLPR short allele (Table 1).

\section{Materials}

Twenty-five negative and 25 positive photos were taken from the International Affective Picture System (IAPS). Twenty-five neutral photos were retrieved from the Internet as well as the IAPS. Positive, negative, and neutral images were selected to be equivalent across conditions on basic low-level image statistics, equated in log luminance $\left(F_{(2,72)}<1\right)$ and RMS contrast $\left(F_{(2,72)}<1\right)$. Positive and negative images were selected to be equivalent in ratings of arousal or emotional salience. Based on ratings by a separate set of participants (Todd et al., 2012), negative, positive, and neutral images did not differ in whether they contained single versus multiple objects $\left(F_{(2,72)}<1, p>0.1\right)$, difficulty of figure ground discrimination (scale of $1-7 ; F_{(2,72)}<1, p>0.5$ ), scene complexity (scale of $\left.1-7 ; F_{(2,72)}<1, p=0.5\right)$, or number of human figures $\left(F_{(2,72)}\right.$ $<1, p>0.6)$.

Gaussian all-color noise was superimposed over each image using Adobe Photoshop 7.0. To minimize variance associated with differences in luminance and contrast across images, the "standard" image used for magnitude estimation was created to match each corresponding "target" image. To create standards that varied as little as possible from paired images in featural characteristics, each image was phase scrambled and overlaid with $10 \%, 15 \%$, or $20 \%$ noise. Both standard and comparison images subtended a visual angle of $13 \times 9.5^{\circ}$.

\section{fMRI procedures}

Localizer task. Our previous research has found that ratings of EEV modulate object-sensitive regions of lateral occipital complex (LOC; Todd et al., 2012). To independently localize category-selective regions of visual cortex, we used a block design task that alternated blocks of line drawings of objects and scrambled line drawings to localize object-selective regions of the LOC_-an early stage in the ventral object-processing stream (GrillSpector et al., 1998). The task also included blocks of faces and places to localize other category-selective regions of visual cortex. Each $20 \mathrm{~s}$ block contained five images presented for $4000 \mathrm{~ms}$ each. Blocks alternated randomly to minimize category predictability with six blocks of each image category. In each block participants performed a one-back task. We further used the contrast between object drawings (easy) versus scrambled drawings (difficult) as a measure of working memory load used to localize frontoparietal regions.

Noise estimation task. We used the visual noise magnitude estimation (NsEst) task to (1) elicit subjective ratings of the perceptual vividness of images overlaid with Gaussian noise and (2) measure trial-by-trial modulation of BOLD activity by behavioral indices of perceptual vividness. In the NsEst task, images and standards were presented in five separate runs of 30 trials. Each image was presented twice at two of three levels of standard noise $(10,15$, and $20 \%)$ for a total of 150 trials (50 negative, 50 positive, and 50 neutral). In each $12 \mathrm{~s}$ trial (Fig. 1) a standard was presented for $1500 \mathrm{~ms}$, followed by a $500 \mathrm{~ms}$ interstimulus interval, followed by the picture, which was presented for $1500 \mathrm{~ms}$. After a randomly jittered interval of $1500 \mathrm{~ms}, 2000 \mathrm{~ms}$, or $2500 \mathrm{~ms}$, the sliding scale response meter appeared for $4000 \mathrm{~ms}$, followed by a randomly jittered intertrial interval of $2000 \mathrm{~ms}, 2500 \mathrm{~ms}$, or $3000 \mathrm{~ms}$. Participants used index and middle fingers to move a cursor along a 14-point sliding scale to rate the degree of noisiness of the image relative to the standard. Ratings ranged from "A lot less noisy" (1) to "A lot more noisy," (14) with "The same as standard" (8) in the center of the scale. Fifty null trials (1/3), consisting of $12 \mathrm{~s}$ of fixation, were included at randomized intervals. Before entering the scanner participants completed a series of 12 practice trials using neutral stimuli.

After data were collected, the following transformations were performed on behavioral responses to the NsEst task to serve as indices of (1) perceptual vividness for behavioral data analysis and (2) EEV for fMRI analysis. First, we mean-centered noise estimation ratings (between 1 and 14) and multiplied them by -1 so they reflected the inverse of noisiness $\left(N s E s t^{-1}\right)$ - a positive measure of vividness. This served as a measure of (1) perceptual vividness for each noise level and emotional category.

$$
\mathrm{NsEst}^{-1}=\left(\mathrm{X}_{\mathrm{NsEst}}-\overline{\mathrm{X}}_{\mathrm{NsEst}}\right) \times(-1)
$$

For item analyses, we calculated a metric of the average perceptual vividness for each image used in the task by averaging NsEst ${ }^{-1}$ for each image across all participants in each genotype group. To generate an $\mathrm{NsEst}^{-1}$ regressor that reflected (2) EEV for fMRI analysis, we further regressed the objective noise level of the standard (StanNoise; less than, same as, or more than the target) on $\mathrm{NsEst}^{-1}$ for each image used in the task. The residuals, or residualized $N s E s t^{-1}\left(\right.$ ResNsEst $\left.^{-1}\right)$, indexed the variance in perceptual vividness associated with emotional salience after controlling for accurate identification of objective noise level. This measure of ResNsEst ${ }^{-1}$ was used as a trial-by-trial parametric modulator in the fMRI analysis.

One week after the scan, participants logged onto a website, where they performed a surprise recognition memory task (not reported here) and rated each image for emotional salience by rating how "emotionally arousing" each image was. Each of the 75 photos was randomly presented without noise. Participants were asked to use a numerical scale from 1 (the image was not emotionally arousing) to 7 (the image was extremely emotionally arousing). 
fMRI acquisition. Imaging data were collected with a 3 Tesla Siemens scanner using a 12-channel head coil. Both the localizer and experimental tasks were programmed in E-prime Version 1.2 (Psychology Software Tools). Stimuli were presented on a rear-mounted projection screen set at a resolution of $1024 \times 758$. For each subject, a 3D MPRAGE was used to acquire a high-resolution T1-weighted structural volume: $\mathrm{TR}=1760$ $\mathrm{ms} ; \mathrm{TE}=2.2 \mathrm{~ms} ; \mathrm{FOV}=256 \times 256$; slice thickness $=1 \mathrm{~mm} ; 176$ slices; total acquisition time $=7: 32 \mathrm{~min}$.

3D field maps (coplanar with the fMRI slices) were acquired on each subject by measuring the phase of non- EPI gradient-echo images at two echo times (Jezzard and Balaban, 1995; Jenkinson, 2003). Parameters for the field mapping series were as follows: TR $=793 \mathrm{~ms}$; TE1 $=5.19 \mathrm{~ms}$ and TE2 $=7.65 \mathrm{~ms}$; flip angle $=60 ; \mathrm{FOV}=211 \mathrm{~mm}$. Thirty-five slices were acquired with a voxel size of $3.3 \times 3.3 \times 3.5 \mathrm{~mm}$. EPI parameters for the two functional tasks were as follows: $\mathrm{TR}=2000 \mathrm{~ms}$; TE $=25 \mathrm{~ms}$; flip angle $=78^{\circ} ; \mathrm{FOV}=211 \mathrm{~mm}$.

Preprocessing. Functional activation was determined from the BOLD signal (Friston et al., 1995) using the software Statistical Parametric Mapping (SPM8; University College London, UK). The first five time points were removed from each functional run to allow for BOLD equilibration. After image reconstruction of the time series, slice-timing correction and motion correction using spatial realignment were performed. We next applied the 3D field maps to unwarp the time series, thereby correcting for EPI image distortions caused by inhomogeneities in the magnetic field and subsequently coregistering individuals' time series with their T1 weighted structural image. The T1 image was bias corrected and segmented using template (ICBM) tissue probability maps for gray/white matter and CSF. Normalization parameters were obtained from the tissue-segmentation procedure and subsequently applied to the time series data (resampling to $3 \mathrm{~mm}^{3}$ voxels). Finally, time series data were smoothed with a $6 \mathrm{~mm}$ full-width half-maximum Gaussian kernel.

First-level statistical models. Procedures were identical to those reported previously (Todd et al., 2012). For each subject, first-level general linear models were applied to localizer data and data from the noise estimation task. Each model included within-session global scaling (default), high-pass filtering to remove low-frequency signal drift (period = $128 \mathrm{~s}$ ), and the AR1 method of estimating temporal autocorrelation.

For the localizer data, boxcar stimulus functions were convolved with the canonical haemodynamic response function (HRF). Conditionspecific regressors were included that modeled objects (line drawings) and scrambled objects as well as faces and places.

For the experimental data, a delta function regressor was modeled for image onset and convolved with the canonical HRF for each trial. To best characterize our behavioral results, we examined trial-by-trial parametric modulation of BOLD by the full range of our behavioral measure of EEV across all emotion categories and objective noise levels. Such an analysis recapitulates the image-by-image item analysis used in the behavioral data, while allowing comparison with our previous findings using this task (Todd et al., 2012). Moreover, ADRA2b may influence sensitivity to low-level perceptual features such as contrast, color, and scene complexity. This design also allowed us to extract contrast files for parametric modulation by EEV after controlling for low-level visual features. We included five parametric modulators for image onsets, described by order of entry into the model as follows: (1) scene complexity, (2) hue, (3) contrast, (4) mean visual saliency, and (5) inverse noise estimation $\left(\mathrm{NsEst}^{-1}\right)$. The inverse noise estimation rating $\left(\mathrm{NsEst}^{-1}\right)$ served as a measure of perceptual vividness. SPM treats the ordering of regressors such that any shared variance is accounted for by the regressors entered first. Thus, this fifth regressor of interest was orthogonalized with respect to the featural salience regressors (1-4). Calculation of regressors 1-4 for each image was as follows. Objective image statistics were computed using the Image Processing Toolbox packaged with MATLAB 7.0. Luminance statistics were derived from the average log luminance (Reinhard et al., 2002). Hue was calculated using MATLAB's rgb2hsv function. Edges were detected using a Canny edge detector with a threshold of 0.5 . Lines were detected by using a Hough transform and the number of detected lines was calculated for each image. Visual salience has been defined as those basic visual properties, such as color, intensity, and orientation, that preferentially bias competition for rapid, bottom-up attentional selection (Itti and Koch, 2001). While visual salience has been used to predict sequential attentional selection of regions within a single image, we derived a measure of mean global saliency for each image to control for visual salience differences between images. To derive image-specific salience magnitudes, visual saliency was computed by averaging the saliency values across all image pixels using the Saliency Toolbox (Walther and Koch, 2006).

ResNsEst ${ }^{-1}$ regressor. Our goal was to compare differences in BOLD response reflecting EEV in each group. As reported below, behavioral data in noncarriers at the group level did not show statistically significant differences between arousing and neutral stimuli, they showed the same direction of response as deletion carriers. Because there was a similar trend in the noncarriers, we expected to be able to pick up on neural differences in noncarriers between the two conditions. Thus, to generate a ResNsEst ${ }^{-1}$ regressor, after averaging NsEst ${ }^{-1}$ across participants image by image, we calculated standardized residual NsEst ${ }^{-1}$ values after controlling for the objective noise level of the standard. We did this for $A D R A 2 b$ deletion carriers and noncarriers separately to probe brain activation specifically associated with the aspect of perceptual vividness linked to emotional salience. The standardized residuals thus served as an index of EEV that could be examined trial by trial across the task. Because this regressor was entered into the regression last, variance shared with low-level features was partialed out and ResNsEst ${ }^{-1}$ accounted for only the unique remaining variance. This approach also allowed us to further examine regions modulated by low-level features.

Second-level statistical models. Regions of interest for key regions specified by previous research and by the BANE model were defined using functional and anatomical templates. The functional localizer task was used to define visual cortex regions expected to be modulated by EEV as well as frontoparietal regions implicated in executive attention. For the localizer task, $T$ contrast files for each condition (object drawings, scrambled objects, and places and faces) from each individual were entered into a one-way ANOVA, with condition as the single factor. The contrast for [objects $>$ scrambled objects] was used to specify shape-selective activation in the LOC. Functionally defined masks were created using $10 \mathrm{~mm}$ spheres around maxima activations in the group maps (MNI coordinates: $51,-76,1$ and $-45,-79,1)$ thresholded at $p<0.05$ (FWE). Similarly, activation in parietal regions associated with the one-back task yielded the center coordinates for the corresponding masks $(-33,-55$, 49 and $36,-55,43$ ) also with a threshold of $p<0.05$ (FWE). Anatomical masks for right and left amygdala and VMPFC were created from automated anatomical labeling (AAL) templates (Tzourio-Mazoyer et al., 2002) based on a spatially normalized high-resolution T1 single-subject dataset using the MarsBaR toolbox (Brett et al., 2002). AAL templates for left rectal gyrus and medial orbitofrontal cortex (OFC) were combined to create the VMPFC mask. Together, these ROIs were used for small volume correction for the noise estimation task.

For the noise-estimation task our primary goal was to examine the relationship between $A D R A 2 b$ genotype and BOLD activation modulated by EEV. A two-sample $t$ test based on the contrast files for ResNsEst $^{-1}$ from the first-level parametric analysis was performed to directly compare $A D R A 2 b$ deletion carriers and noncarriers. Although our primary focus was on $A D R A 2 b$, because of group differences in the ratio of $5 H T T L P R$ short allele carriers to noncarriers, we wanted to statistically control for the potential influence of $5 H T T L P R$ on our results. Secondarily, we also wanted to examine potential main effects and interactions with ADRA2b. Thus, 5HTTLPR was included as a covariate in the grouplevel analysis. As the distribution of COMT genotype was equal across $A D R A 2 b$ groups, and preliminary analyses revealed no effects of COMT, COMT was not included in this analysis. Results of the omnibus test for this analysis, thresholded at $p<0.05$ after controlling for FWE, are reported in Table 2.

Path analysis. To further probe results of our voxelwise examination of EEV-related activation, we next used path analysis to examine potential coactivation patterns between regions associated with trial-by-trial subjective ratings of EEV. This approach was optimal for examining results of our parametric modulation because, unlike other approaches to modeling coactivation patterns (e.g., dynamic causal modeling), it did not require a full factorial design. To extract time series information for the 
Table 2. fMRI omnibus test results

\begin{tabular}{|c|c|c|c|c|c|c|c|c|}
\hline Brain region & $B A$ & $x$ & $y$ & $z$ & Voxels $^{a}$ & $F$ & $z$ & $p$ \\
\hline Left middle occipital gyrus & 19 & -48 & -79 & 4 & 81 & 20.28 & 5.61 & 0.001 \\
\hline Right inferior occipital gyrus & 19 & 45 & -76 & -5 & 52 & 19.17 & 5.49 & 0.001 \\
\hline Right Fusiform/parahippocampal gyrus & 30 & 30 & -40 & -8 & 450 & 18.55 & 5.43 & 0.001 \\
\hline Left middle orbitofrontal/rectal gyrus & 11 & -12 & 32 & -11 & 52 & 17.62 & 5.32 & 0.003 \\
\hline Left fusiform gyrus & 37 & -30 & -49 & -8 & 245 & 15.52 & 5.04 & 0.011 \\
\hline
\end{tabular}

${ }^{a}$ Cluster size at $p<0.001$ uncorrected. Regions parametrically modulated by emotionally enhanced vividness (EEV) by genotype after controlling for objective salience; $x, y, z$, coordinates are in MNI space. $P$ values are FWE corrected for multiple comparisons.

path analysis, a separate first-level analysis including six regressors for emotion category (positive, negative, and neutral) and noise level (10, 15, and $20 \%$ noise) was performed. The delta function regressors were used to model stimulus onset for emotion category and standard noise level. Unadjusted signal was extracted from the first eigenvariate of a $3 \mathrm{~mm}$ radius spherical volume of interest (VOI). The VOI was centered on the coordinates of the local maximum within an anatomically bounded region defined by AAL templates for left amygdala and VMPFC, respectively. Peak activation in these regions was based on the first-level contrast [emotionally arousing $>$ neutral]. For the functional ROI "left LOC," the sphere was centered on the coordinates of the local maximum $(-51,-67,16)$ resulting from the second-level analysis of the functional localizer. To obtain comparable time series data, ResNsEst ${ }^{-1}$ was convolved with the hemodynamic response function. Both ROI time series data and ResNsEst ${ }^{-1}$ ratings were averaged separately for the two $A D R A 2 b$ variants at each time point. We thus obtained time point by time point ResNsEst ${ }^{-1}$ ). The SPSS add-on module AMOS (IBM SPSS Amos, Version 21.0) was used to confirm the results and to obtain different indices for model fit.

Model fit can be assessed by various fit indices. The parsimony goodness-of-fit index (PGFI) calculates the proportion of variance accounted for by the estimated population covariance; hence, better model fit is indicated by larger values (Mulaik et al., 1989). The standardized root mean square residual (SRMR), with values smaller than 0.05 indicating good fit (Byrne, 1998), is based on the square root of the difference between the residuals of the sample covariance matrix and the hypothesized covariance model (Hooper et al., 2008). The corrected Akaike information criterion $(\mathrm{AICc})$ is a relative goodness-of-fit index considering model complexity and allowing direct comparison of models. Smaller AICc values are an indicator for a better fit (Akaike, 1974). Finally, the most commonly used index is $\chi^{2}$, which relies on the difference between sample and model covariance matrix (Hu and Bentler, 1999). A small $\chi^{2}$ and a $p$ value bigger than 0.05 suggest a good fit of the model (Barrett, 2007). We report all of these values in the results of the model comparison below.

\section{Genotyping}

Genotyping was performed by the Neurogenetics Laboratory at the Centre for Addiction and Mental Health in Toronto, Canada. For this purpose, a $2 \mathrm{ml}$ sample of saliva had been collected from each participant using an Oragene OG-500 DNA kit (DNA Genotek). DNA was extracted as per manufacturer's instructions and diluted to $20 \mathrm{ng} / \mu \mathrm{l}$ working concentration.

For the ADRA2b 9 bp deletion locus, total genomic DNA (60 ng) was combined with $1 \times$ MBI Fermentas PCR buffer containing $\mathrm{KCl}, 1.5 \mathrm{~mm}$ $\mathrm{MgCl}_{2}$ (MBI Fermentas), $0.0325 \mu \mathrm{g}$ of each primer (forward primer sequence: $5^{\prime}$ HEX-CAGAAGGAGGGTGTTTGTGG; reverse primer sequence: 5' CCACTGCCCACCTATAGCAC), $0.2 \mathrm{~mm}$ each dNTP (MBI Fermentas), and 0.6 U Taq polymerase (MBI Fermentas) to a total volume of $15 \mu \mathrm{l}$ in a 96-well PCR plate. The PCRs were subjected to an initial denaturation for $5 \mathrm{~min}$ at $95^{\circ} \mathrm{C}$, followed by 30 cycles of amplification in an $\mathrm{AB} 2720$ thermal cycler: denaturing for $30 \mathrm{~s}$ at $95^{\circ} \mathrm{C}$, annealing for $30 \mathrm{~s}$ at $60^{\circ} \mathrm{C}$ and extension for $30 \mathrm{~s}$ at $72^{\circ} \mathrm{C}$, and a final extension at $72^{\circ} \mathrm{C}$ for 10 min. Similarly for the SLC6A4 LPR (5HTTLPR), $40 \mathrm{ng}$ total genomic DNA was combined with $1 \times$ MBI Fermentas PCR buffer containing $\left(\mathrm{NH}_{4}\right)_{2} \mathrm{SO}_{4}, 1.5 \mathrm{~mm} \mathrm{MgCl}$ (MBI Fermentas) $0.0325 \mu \mathrm{g}$ of each primer (forward primer labeled with 5' HEX fluorescent tag), $0.16 \mathrm{~mm}$ each dNTP (MBI Fermentas), and 1 U Taq polymerase (MBI Fermentas) to a total volume of $25 \mu \mathrm{l}$ in a 96-well PCR plate. The PCRs were subjected to an initial denaturation for $3 \mathrm{~min}$ at $95^{\circ} \mathrm{C}$, followed by 40 cycles of amplification in an Eppendorf Mastercycler Pro $\mathrm{S}$ thermal cycler: denaturing for $30 \mathrm{~s}$ at $95^{\circ} \mathrm{C}$, annealing for $30 \mathrm{~s}$ at $61^{\circ} \mathrm{C}$ and extension for $1 \mathrm{~min}$ at $71^{\circ} \mathrm{C}$, and a final extension at $72^{\circ} \mathrm{C}$ for $10 \mathrm{~min}$. Five microliters of the PCR product was combined with $1 \times$ New England BioLabs Buffer 2 and $10 \mathrm{U}$ MspI restriction enzyme (New England BioLabs) in a total volume of 30 $\mu \mathrm{l}$ was digested overnight at $37^{\circ} \mathrm{C}$. For both $A D R A 2 b$ and the LPR, the final products were electrophoresed on an AB 3130-Avant Genetic Analyzer as per manufacturer's directions, and product sizes determined by comparison to GeneScan 500 ROX size standard using GeneMapper (version 4.0).

For COMT, an SNP was genotyped using TaqMan predesigned assays (Life Technologies): Val158Met in the COMT gene (rs4680; assay ID C_25746809_50). Twenty nanograms of genomic DNA were amplified as per manufacturer's directions scaled to a total volume of $10 \mu \mathrm{l}$ in an $\mathrm{AB}$ 2720 thermal cycler. Postamplification products were analyzed on the ABI Prism 7500 Sequence Detection System using the allelic discrimination option, and genotype calls were determined manually by comparison to six No Template Controls.

Genotyping of $10 \%$ of samples from each run was replicated for quality control purposed for each marker.

\section{Results}

For all analyses, based on previous research (de Quervain et al., 2007; Rasch et al., 2009), homozygote and heterozygote ADRA2b deletion carriers were treated as a single group due to the low number of homozygotes. Similarly, homozygous and heterozygous carriers of the 5HTTLPR short allele (Canli and Lesch, 2007) and the COMT val allele were also treated as a single group. Twenty-two participants carried the 5HTTLPR short allele and 17 were homozygous long allele carriers; 27 carried the COMT val allele and 12 were homozygous met carriers.

\section{Behavioral results}

\section{Noise estimation task}

Because our focus was on EEV as our dependent measure, we first calculated NsEst ${ }^{-1}$ as a measure of perceptual vividness in each condition. Thus NsEst ${ }^{-1}$ served as an index of the vividness of the signal of the underlying image in relation to the overlaid noise. To examine genotype-related differences in $\mathrm{NsEst}^{-1}$ related to the emotional salience of the stimulus and the objective level of standard noise, we performed a three-way repeatedmeasures ANOVA with emotion category (negative, positive, and neutral) and standard noise level $(10,15$, and 20\%) as withinsubject factors. Genotype groups (ADRA2b, 5HTTLPR, and $C O M T$ ) were between-subject factors. All results were Greenhouse-Geisser corrected for violations of sphericity when necessary and contrasts were Bonferroni corrected for multiple comparisons.

\section{Within-subject effects}

Results revealed a main effect of noise level $\left(F_{(2,66)}=76.05, p<\right.$ $\left.0.001, \eta_{p}^{2}=0.70\right)$. Linear contrasts revealed that participants accurately rated the comparison pictures as less noisy relative to the 
a

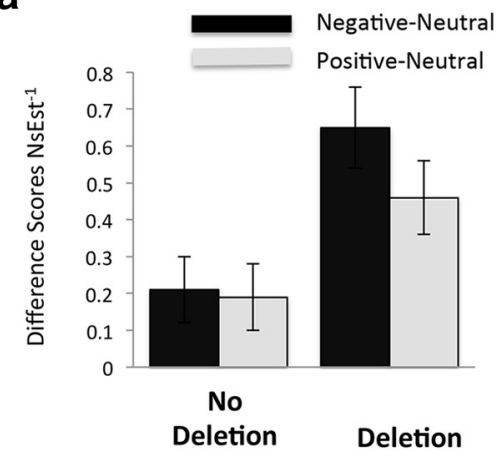

b

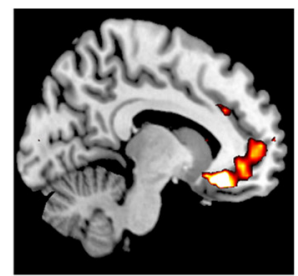

$-12 \quad 26-14$

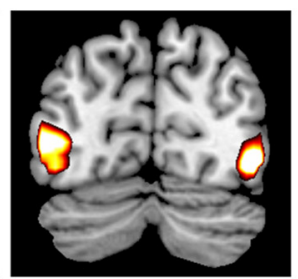

$-48-73-7$

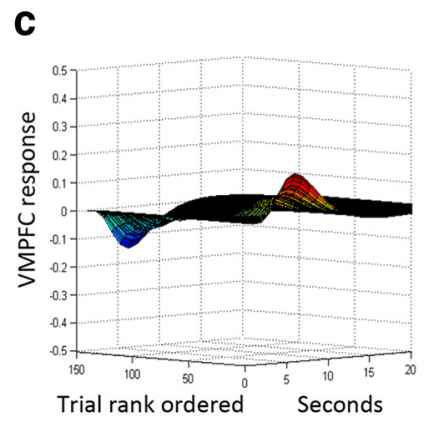

No Deletion

d

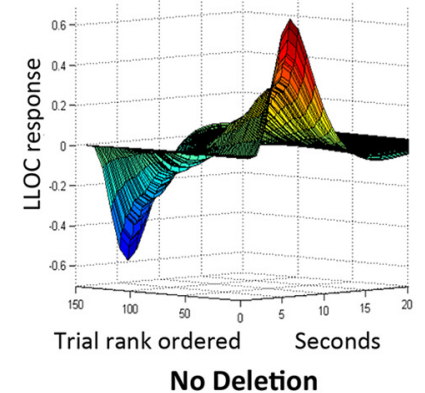

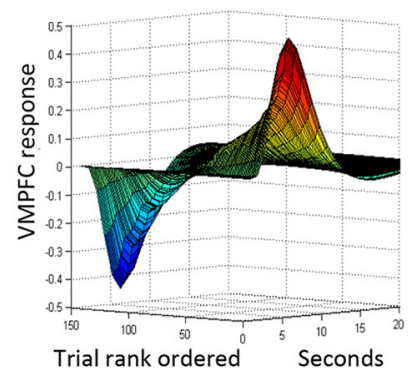

Deletion

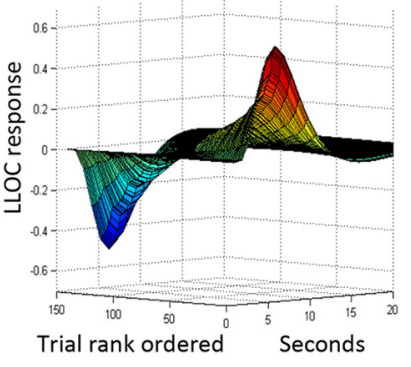

Deletion

Figure 3. The influence of $A D R A 2 b$ on behavioral and neural measures of emotionally enhanced vividness (EEV). $\boldsymbol{a}$, Difference scores for ratings of inverse noise estimation (NsEst ${ }^{-1}$ ) for negative and positive $>$ neutral stimuli in noncarriers and carriers of the ADRA2b deletion variant. Deletion carriers show greater EEV than noncarriers. $\boldsymbol{b}$, Statistical maps showing parametric modulation by EEV in the ventromedial prefrontal cortex (VMPFC) for ADRA2b carriers $>$ noncarriers, and in the lateral occipital complex (LOC) showing modulation by EEV across both groups ( $n=37)$. $\boldsymbol{c}, \boldsymbol{d}$, Illustration of trial-by-trial modulation of VMPFC ( $\boldsymbol{c}$ ) and left LOC (LLOC; $\boldsymbol{d})$ by EEV over the time course of the hemodynamic response for ADRA2b deletion carriers $(n=21)$ and noncarriers $(n=18)$. The trial axes are rank ordered (from right to left) from highest (1) to lowest (150) ratings of EEV.

standards with increasing standard noise levels $\left(F_{(1,33)}=82.12\right.$, $p<0.001)$. Thus, participants were reliably sensitive to differences in levels of objective noise. There was also a main effect of emotion category $\left(F_{(2,66)}=21.36, p<0.001, \eta_{p}^{2}=0.39\right)$. Despite containing identical levels of noise, contrasts showed that both positive and negative images were perceived as more perceptually vivid relative to the standards than neutral images $\left(F_{(1,33)}=\right.$ $39.89, p<0.001)$. Negative and positive pictures were rated as more vivid than neutral pictures at all noise levels $(p s<0.05)$. Vividness ratings for positive and negative images did not differ from each other at any noise level ( $p s>0.9)$. There was also an interaction between noise and emotion category $\left(F_{(4,132)}=3.67\right.$, $\left.p=0.007, \eta_{p}^{2}=0.10\right)$, showing the main effects reported above to be greatest at the lowest level of standard noise and reducing with standard noise level. Whereas vividness ratings for both positive and negative images were higher than for neutral images at noise levels 1 and 2, at noise level 3 only negative images were rated as more vivid than neutral ( $p s<0.05$ ). Thus, although noise estimation was overall accurate, there was a pronounced effect of $\mathrm{EEV}$, with participants rating emotionally salient images as less noisy, or more perceptually vivid, than neutral images. This effect, which replicates previous findings (Todd et al., 2012), was more pronounced at lower levels of standard noise.

Between-subject effects

There was a main effect of $A D R A 2 b\left(F_{(1,33)}=4.51, p=0.04, \eta_{p}^{2}=\right.$ 0.12 ), showing that deletion carriers rated all images as noisier, or overall lower in vividness than noncarriers. Crucially, the main effect of $A D R A 2 b$ was qualified by an interaction with emotion category $\left(F_{(2,66)}=6.49, p=0.003, \eta_{p}^{2}=0.16\right.$; Fig. $\left.3 a\right)$, with deletion carriers showing more of an effect of EEV than noncar- riers. That is, deletion carriers indicated higher levels of perceptual vividness for negative and positive relative to neutral images than noncarriers, and contrasts between emotionally salient and neutral images were significant in deletion carriers $(p s<0.05)$ but not noncarriers $(p s>0.2)$. Aside from $A D R A 2 b$, there were no significant main effects of genotype, and there were no interactions with emotional category or noise level $(p s>0.15)$ related to COMT or 5HTTLPR genotype ( $p$ s $>0.18$ ). In summary, as predicted, deletion carriers showed greater effects of emotion category on noise estimation ratings than noncarriers, indicating higher levels of EEV. Thus, the extent to which emotionally salient relative to neutral pictures are experienced as more perceptually vivid differs across individuals and is dependent on genetic variation linked to NE.

\section{Arousal ratings}

Arousal ratings were missing from two participants who failed to log on 1 week later (one deletion carrier and one noncarrier). A three-way repeated-measures ANOVA was performed on arousal ratings with emotion category (negative, positive, and neutral) as the within-subject factor and genotype group (ADRA2b, $5 H T T L P R$, and COMT) as between-subject factor. Results showed a main effect of emotion category $\left(F_{(2,60)}=126.63, p<\right.$ $\left.0.001, \eta_{p}^{2}=0.81\right)$. Ratings for each emotion category differed from each of the others, with highest arousal ratings for negative images $($ mean $=4.72)$, next highest for positive images $($ mean $=$ 4.02 ), and lowest for neutral images (mean $=1.55$; $p$ s $<0.005$ ). There were no main effects of genotype or interactions between genotype and emotion ( $p s>0.24$ ). Thus while arousal ratings reflected normalized ratings of the stimulus categories, subjective ratings of arousal did not differ by genotype group. 
Item analyses

In a further step, we performed an item analysis to ascertain the influence of emotional salience on perceptual vividness, image by image, in each group separately. For this analysis each measure of interest, including measures of emotional salience, noise estimation, and measures of the low-level features of each image, was averaged across participants for each image used in the experiment. To obtain our emotional salience measure, we calculated the mean emotional arousal ratings for each image from each participant and then averaged this value across participants for each image. To obtain a measure indexing perceptual vividness we calculated NsEst ${ }^{-1}$ (i.e., the inverse of the meancentered noise estimation). As measures of featural salience, we obtained the metrics for each image, including number of edges, hue, and a global computational metric of visual salience (Itti and Koch, 2001). Separate correlation analyses in $A D R A 2 b$ carriers and noncarriers revealed that, image by image, mean arousal ratings and NsEst ${ }^{-1}$ were significantly correlated in deletion carriers $(r=0.34, p=0.003)$. The correlation between arousal and NsEst $^{-1}$ was positive, but nonsignificant in noncarriers $(r=$ $0.18, p=0.13)$. There was a significant correlation between NsEst $^{-1}$ and visual salience for deletion carriers $(r=0.26, p=0.01)$ and for noncarriers $(r=0.34, p=0.001)$, indicating that within each group, greater perceptual vividness was also influenced by low-level featural salience. In a next step, hierarchical multiple regressions were performed for each group. Measures of featural salience (hue, scene complexity, and visual salience) were entered at the first level. Arousal ratings (emotional salience) were entered at the second level. Results revealed that, after controlling for low-level features, emotional salience predicted NsEst ${ }^{-1}$ in deletion carriers $\left(R^{2} \Delta=0.05, p=0.04\right)$. Emotional salience did not significantly predict NsEst ${ }^{-1}$ in noncarriers $\left(R^{2} \Delta=0.01, p=\right.$ 0.39 ). In contrast, featural salience contributed to perceptual vividness for both deletion carriers $\left(R^{2} \Delta=0.11, p=0.04\right)$ and noncarriers $\left(R^{2} \Delta=0.14, p=0.02\right)$. Thus, item analyses performed for each group separately revealed that featural salience had similar effects on perceptual vividness in both $A D R A 2 b$ carriers and noncarriers. In contrast, only deletion carriers demonstrated a reliable influence of emotional salience on perceptual vividness.

Although examination of the effects of $A D R A 2 b$ on emotional enhancement of memory (EEM) was not the focus of the present study, for continuity with previous research we did examine the influence of $A D R A 2 b$ on EEM. Analysis of memory vividness ratings and recognition memory accuracy revealed no influence of $A D R A 2 b$ on overall memory or the effect of emotion on memory $(p s>0.60)$.

\section{fMRI results}

Unless otherwise specified, all results are reported with FWE correction for multiple comparisons either at the whole-brain level or with small-volume correction within prespecified masks. All $x$, $y, z$ coordinates are in MNI space.

Omnibus effects of genotype on modulation by EEV

Contrast files for ResNsEst ${ }^{-1}$, indexing the trial-by-trial modulation of BOLD activity by EEV, after controlling for the contribution of low-level features of each image, were entered into a two-sample $t$ test with ADRA2b deletion carriers and noncarriers as independent groups. The 5HTTLPR genotype was included as a covariate. Regions significantly activated within this model at a threshold of $p<0.001$, corrected for FWE, are summarized in Table 2. Specific contrasts characterizing activation at each locus are discussed below.
Effects of ADRA2b genotype on modulation by EEV

Contrasts revealed that for both $A D R A 2 b$ carriers and noncarriers, activation in the left LOC $[(-48,-73,7) ; t=5.69, z=4.76$, FWE $p<0.001 \mathrm{svc}$ ], was modulated by ResNsEst ${ }^{-1}$. The same pattern was observed in right $\operatorname{LOC}[(54,-70,7) ; t=3.86, z=$ 3.50 , FWE $p=0.02 \mathrm{svc}$. Thus, after controlling for featural salience and 5HTTLPR allele, greater ratings of EEV were reflected in greater LOC activation in both groups (Fig. $3 b$ ). This result replicates our previous finding of enhanced LOC activity with higher levels of ResNsEst ${ }^{-1}$, suggesting that the subjective experience of perceptual vividness, or enhanced seeing, is linked to greater activation in object-sensitive regions of the visual cortex. Importantly, it further indicates that this aspect of EEV is similarly mediated by visual cortex activity in both $A D R A 2 b$ deletion carriers and noncarriers alike. To a lesser degree, EEV modulation in our a priori-defined left amygdalar region was also observed across all participants $[(-24,-1,-23) ; t=1.98, z=1.92$, $p=0.03$ uncorrected], although contrasts revealed that activation in the left amygdala was greater for deletion carriers than noncarriers $[(-27,-1,-23) ; t=2.01, z=1.95, p=0.03$ uncorrected].

In contrast, activity in the VMPFC unambiguously differentiated $A D R A 2 b$ deletion carriers from noncarriers (Fig. $3 b, d$ ): Deletion carriers showed greater positive modulation by EEV than noncarriers in the left VMPFC, with a peak activation in the rectal gyrus $[(-12,29,-14) ; t=3.84, z=3.48$, FWE $p=0.048 \mathrm{svc}]$. Thus, emotional enhancement of vividness was associated with greater VMPFC signal in deletion carriers versus noncarriers. Activation in the right intraparietal sulcus (IPS) $(33,-58,46)$ activated by the attention localizer showed the opposite pattern: activation was negatively associated with ResNsEst ${ }^{-1}$ for deletion carriers but not for noncarriers $(t=3.81, z=3.46$, FWE $p=0.02 \mathrm{svc})$. In deletion carriers, activity in the IPS, associated with executive attention, decreased when EEV increased. In summary, the $A D R A 2 b$ polymorphism-differentiated brain activity correlated with EEV in two regions. Deletion carriers showed greater activity in the VMPFC, a key BANE node implicated in salience evaluation, relative to noncarriers. At the same time, deletion carriers also showed negatively correlated activation in a parietal region mediating executive attention, suggesting fewer executive resources were recruited in trials that were higher in EEV.

As tonic noradrenergic activity is sensitive to salience in general (Aston-Jones and Cohen, 2005), including low-level visual salience, we performed a follow-up analysis on activity modulated by the regressor for image contrast included in the same first-level model. We investigated image contrast because it is a feature we have previously found to evoke activity in the amygdala and visual cortices. Results showed that VMPFC activity was more sensitive to contrast in deletion carriers than noncarriers $[(0,41,-20) ; t=3.79, z=3.46$, FWE $p=0.03 \mathrm{svc}]$, indicating an influence of $A D R A 2 b$ on sensitivity to low-level visual salience in this region as well. It should be noted, however, that the VMPFC modulation by EEV we reported was based on first-level contrasts in which variance due to low-level features was partialed out. Thus these contrasts reflected variance that was unique to EEV.

\section{Control analysis: effects of 5HTTLPR genotype on modulation by EEV}

Here we further examined contrasts indexing effects of the covariate modeling $5 H T T L P R$ genotype, focusing on regions where modulation by ResNsEst ${ }^{-1}$ was greater for short allele carriers, as well as contrasts modeling interactions between 5HTTLPR and 
a

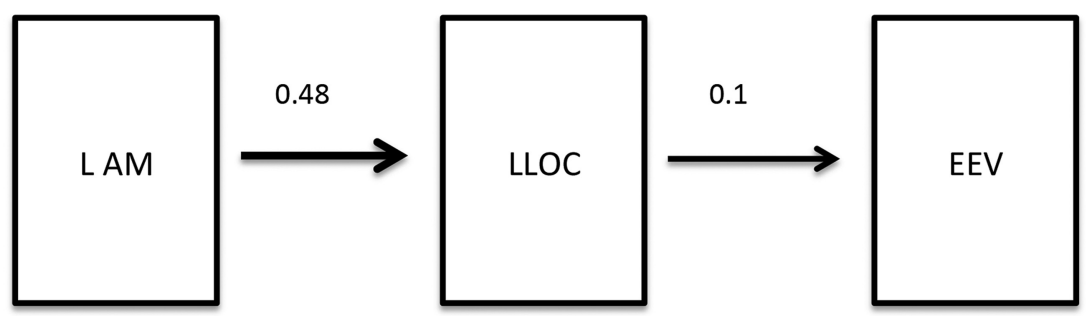

b

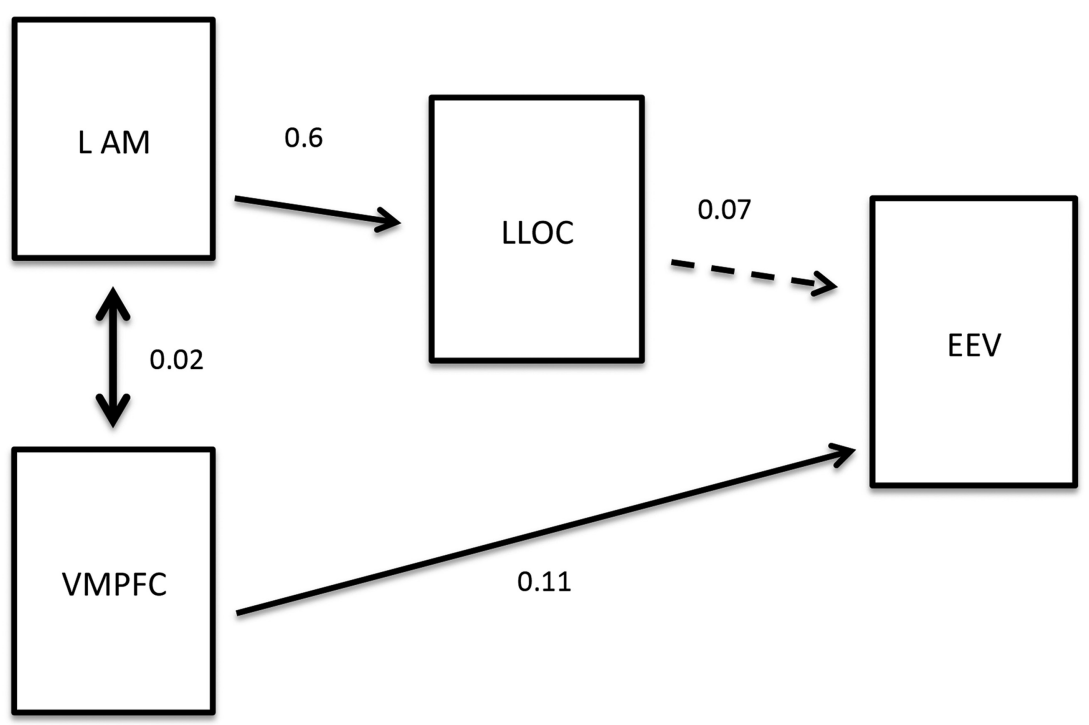

Figure 4. $\quad \boldsymbol{a}$, Parsimonious model predicting emotionally enhanced vividness (EEV) in noncarriers ( $n=18$ ) of the ADRA2b polymorphism by left lateral occipital complex (LLOC) activity mediated by the left amygdala (LAM). $\boldsymbol{b}$, Complex model predicting EEV in deletion carriers $(n=21)$ of the ADRA2b polymorphism. The dual-route model demonstrates that the left amygdala mediates the effect of LLOC on EEV and simultaneously ventromedial prefrontal cortex (VMPFC) contributes to EEV. For both models, $\beta$-estimates for each path are shown. Significant paths are indicated by solid lines, dashed lines indicate nonsignificance. Bidirectional arrows between left amygdala and VMPFC indicate covariance of these regions, which exhibited a high level of correlated activity $(r=0.46, p<0.001)$, and the covariance statistic indicating the relation between the two variables is shown.

ADRA2b. Modulation by ResNsEst ${ }^{-1}$ was greater for short allele carriers in the left VMPFC $[(-12,32,-14) ; t=6.12, z=5.01$, FWE $p<0.001 \mathrm{svc}]$, as well as in the right amygdala $[(33,-7$, $-14) ; t=3.59, z=3.29$, FWE $p=0.02 \mathrm{svc}$ ]. Thus, carrying the short allele of $5 H T T L P R$ was linked to enhanced EEV modulation in the same region of left VMPFC as carrying the ADRA2b deletion variant; however, in short allele carriers amygdala modulation by EEV was in the right hemisphere. It should be noted that the results we report for $A D R A 2 b$ are significant after accounting for the variance due to $5 H T T L P R$.

\section{Interactions}

Although our sample size was too small to reliably test gene $\times$ gene interactions, exploratory examination of potential interactions revealed a cluster in the left VMPFC $[(-12,32,-14) ; t=$ $5.95, z=4.91$, FWE $p<0.001 \mathrm{svc}]$. This cluster showed a pattern of interaction between ADRA2b and 5HTTLPR genotype, such that there was greater modulation by EEV for noncarriers of the $A D R A 2 b$ deletion variant that carried the short 5HTTLPR allele than deletion carriers who carried the short 5HTTLPR allele.

\section{Path analysis}

The models selected for path analysis focused on statistical influences among regions that have been implicated in EEV and are indicated by the BANE model, which emphasizes reciprocal in- teractions between the LC/NE system and the amygdala, VMPFC, and visual cortices in enhancing attention to affectively salient stimuli (Markovic et al., 2014). The amygdala is a key target site for the NE system and is rich in NE receptors (Jones and Moore, 1977). Indirect modulation of visual cortex activity by NE can be observed via LC modulation of VMPFC and amygdala activity, which in turn influences visual cortex activity (Waterhouse et al., 1990; Gallagher and Holland, 1994; Roozendaal et al., 2009). Finally, the mediating role of the amygdala and the contribution of the left LOC to EEV have been demonstrated previously (Todd et al., 2012), and the results of the parametric modulation further revealed differential VMPFC activation by $A D R A 2 b$ genotype.

We thus focused on two competing path-analysis models to specify how behavioral ratings of EEV may be influenced by the contribution of VMPFC to previously modeled LOC and amygdala activity in carriers and noncarriers of the ADRA2b deletion variant: a parsimonious model in which the influence of the left LOC on $\mathrm{EEV}$ is directly modulated by the left amygdala, and a second model which is an extension of the simple model that adds a direct path from VMPFC to EEV. Because of limitations in imaging the human LC (Astafiev et al., 2010), we did not include the LC in either model.

The parsimonious model (Fig. 4a) revealed a fit for both deletion carriers $\left(\chi^{2}(2)=1.08, p=0.60, \mathrm{SRMR}=0.0159\right.$, PGFI $=0.166$, AICc $=36.62)$ and noncarriers $\left(\chi^{2}(2)=0.301, p=0.58\right.$, SRMR $=$ 0.0092 , PGFI $=0.167$, AICc $=35.85)$. As the overall model fit suggests, activation of the left amygdala predicted left LOC activity (deletion: $b=0.6, p<0.001$; no deletion: $b=0.318$, $p<0.001$ ), which in turn predicted EEV (deletion: $b=0.051$, $p=0.023$; no deletion: $b=0.05, p=0.039)$ for both genotypes. This suggests that the amygdala plays a key role in the emotional modulation of perceptual vividness regardless of NE-related genotypic variation.

The second model (Fig. $4 b$ ) models the high level of covariance between amygdala and VMPFC activity $(r=0.46, p<$ $0.001)$. This dual-route model did not predict EEV adequately in noncarriers $\left(\chi^{2}(1)=10.63, p=0.005\right.$, SRMR $=0.041$, PGFI $=$ $0.198, \mathrm{AICc}=67.50)$. In contrast, the time series data of deletion carriers fit the model well $\left(\chi^{2}(1)=5.64, p=0.06\right.$, SRMR $=$ 0.026 , PGFI $=0.199$, AICc $=62.52)$. The model implicates that, in deletion carriers, the amygdala $(b=0.51, p<0.001)$ mediates the influence of left LOC on EEV $(b=0.033, p=0.148)$; however, in addition, VMPFC directly influences EEV $(b=0.047, p=$ $0.018)$, even when left amygdala and LOC serve as covariates $(b=$ $0.019, p<0.001)$. In this model this pathway accounted for more of the variance in EEV than the amygdala/LOC pathway. Thus, the two-path model best describes the contribution of brain regions to EEV in deletion carriers. 
Amygdala response to arousal

Finally, to link the present findings to previous studies, we used the factorial model reported above to examine differences in amygdala activation in $A D R A 2 b$ carriers relative to noncarriers for negative relative to neutral images. Contrast files for negative $>$ neutral trials revealed activation in the left amygdala $[(-15,-4,-17) ; t=1.89, z=1.85, p=0.03$ uncorrected], showing a similar pattern to those previously reported (Rasch et al., 2009) but only at an uncorrected threshold. Differences in the size of the effect may reflect lower power due to the smaller sample size used in this study. Differences in the extent of the effect may also be due to differences in the experimental task (our task required cognitive distraction from affective content, whereas the study by Rasch et al., 2009 required explicit focus on valence and arousal).

\section{Discussion}

Behavioral results showed that, as predicted, carriers of the $A D R A 2 b$ deletion variant showed higher levels of subjectively experienced perceptual vividness for emotionally salient images (EEV) than noncarriers. Deletion carriers perceived decreased magnitude of Gaussian noise embedded in emotionally arousing images, indicating more vivid perceptual experience of the underlying image, and demonstrated greater coupling between reported emotional arousal and perceptual vividness.

$A D R A 2 b$ deletion carriers have been found to have greater emotional enhancement of memory and susceptibility to intrusive memories following trauma (de Quervain et al., 2007), as well as higher levels of amygdala activation at encoding (Rasch et al., 2009; Cousijn et al., 2010). Our previous research has found that deletion carriers show enhanced attentional tuning to emotionally salient stimuli (Todd et al., 2013), and a stronger link between perceived arousal of stimuli at encoding and subsequent memory (Todd et al., 2014). In the present study ADRA2b deletion carriers showed greater effects of EEV than noncarriers. These behavioral findings suggest that naturally occurring differences in NE receptor function underlie individual differences in the vividness with which we perceive emotionally relevant features of the environment.

fMRI results revealed that both deletion carriers and noncarriers showed modulation of LOC activation by EEV, suggesting that for both groups the experience of enhanced perceptual vividness is linked to enhanced activation in regions of visual cortex associated with object perception and extraction of semantic content (Todd et al., 2012). In contrast, in the VMPFC, greater EEVrelated activation was found in deletion carriers than noncarriers. The finding that greater VMPFC activation modulates EEV for $A D R A 2 b$ deletion carriers is consistent with the proposal that, in humans, NE plays a role in modulating affective biases in perceptual encoding via activation in important nodes of the BANE network (Markovic et al., 2014). The findings of strong group differences in activation in VMPFC support the hypothesis that enhanced EEV is supported by a partially distinct mechanism in noncarriers.

Anatomical data indicate that the VMPFC is closely connected to regions associated with internally generated states rather than those appraising features of the external world (Andrews-Hanna et al., 2010). VMPFC is also linked to regions sensitive to appraisal of stimuli such as the amygdala and more lateral OFC (Carmichael and Price, 1996; Price et al., 1996; Rempel-Clower and Barbas, 1998; Barbas et al., 1999; Cavada et al., 2000; Croxson et al., 2005). A recent study using multivoxel pattern analysis (MVPA) in humans reported population activity in VMPFC that was sensitive to both positive and negative stimuli and independent of stimulus modality, reflecting a more subjective quality of affect (Chikazoe et al., 2014). These data suggest that, whereas the amygdala plays a role in appraising the salience of features of the external world, the VMPFC is more involved in aspects of emotional evaluation and experience that are internally generated.

Deletion carriers also showed greater EEV-related decreases in IPS, a key region in frontoparietal control networks, than in noncarriers. IPS activity specifically modulates executive aspects of working memory (Rottschy et al., 2012; Nee et al., 2013) and increases with attention to visual detail (Guerin et al., 2012). We previously found this region to be negatively associated with EEV, suggesting increased task-related visual attention to images experienced as less perceptually vivid, and indicating a trade-off between attentional executive and affective salience networks (Todd et al., 2012). The present findings suggest that this tradeoff can be observed in ADRA2b deletion carriers only, suggesting that individual differences related to NE receptor activity play a role in this interaction between perceptual and attentional systems.

The BANE model (Markovic et al., 2014) emphasizes the role of NE in tuning valuation systems mediating enhanced encoding of emotionally salient aspects of the world. A salient stimulus activates LC neurons, which project widely to cortical and subcortical regions (Sara, 2009; Sara and Bouret, 2012). In addition to directly altering the gating and tuning of neuronal activity in the visual cortex, the LC modulates visual cortex activity indirectly via the amygdala and prefrontal cortices (Waterhouse et al., 1990; Gallagher and Holland, 1994; Roozendaal et al., 2009). Descending influences from both the amygdala and OFC/VMPFC provide information about contextually determined relevance (Aston-Jones and Cohen, 2005), which can then modulate the pattern of LC firing to reflect salience within the given context (Fig. 2).

The goal of our path analysis was to elaborate on the trial-bytrial modulation of the BOLD response by subjective ratings of EEV that reflected our behavioral findings. This analysis supported a model in which deletion carriers recruit additional nodes of valuation networks emphasized in the BANE model. It suggests that, for noncarriers, a simple model by which amygdala activation modulates the influence of visual cortex activation on EEV explains the data better than a more complex model that includes the VMPFC. Thus, for noncarriers, tagging of stimulus salience by the amygdala may heighten activity in the LOC that is directly related to processing of the visual stimulus. This in turn results in the heightened experience of perceptual vividness. In contrast, for deletion carriers the data were better explained by a model that included a second pathway, by which VMPFC directly influenced behavior, which accounted for a larger proportion of the variance in behavior than the amygdala/LOC pathway. One interpretation is that, for deletion carriers, the emotionally tuned vividness of perception is more strongly informed by internally generated affective experience. We note that, based on this anal$y$ sis, it is not possible to infer the causal influence of activity in one region on another as we could with other methods such as dynamic causal modeling (Wang et al., 2014).

Although they showed greater EEV, ADRA2b deletion carriers rated all stimuli as higher in overall noise than noncarriers. One explanation is that tonic NE activity, thought to be influenced by $A D R A 2 b$, is sensitive to salience in general (Aston-Jones and Cohen, 2005), including low-level visual salience. It is possible that deletion carriers are more perceptually sensitive to visual salience, resulting in greater sensitivity to the task-relevant overlaid 
noise, especially when stimuli are not emotionally salient. Indeed our follow-up analysis revealed that deletion carriers showed greater VMPFC activity in response to contrast. Nonetheless, the EEV results we report here showed activation that passed threshold after controlling for variance from visual salience. Thus, in deletion carriers, sensitivity to affective salience influenced VMPFC activation over and above variance accounted for by sensitivity to low-level salience. Thus, whereas deletion carriers may show greater VMPFC sensitivity to low-level visual features, they also show greater VMPFC sensitivity to EEV.

In this sample we found no effect of $A D R A 2 b$ on emotional modulation of memory. This may reflect insufficient power to detect an effect. Previous smaller- $N$ imaging studies have similarly failed to find effects of $A D R A 2 b$ on emotional enhancement of memory (Rasch et al., 2009).

\section{$5 H T T L P R$}

Although 5HTTLPR has been implicated in affective biases in attention (Canli, 2008), it did not influence behavioral indices of EEV. This is consistent with our previous studies in which carrying the 5HTTLPR short allele failed to predict affective biases in perceptual encoding or links between encoding and memory (Todd et al., 2013). However, in the present study we did not have sufficient power to detect any but the strongest behavioral results. Importantly, carrying the short 5HTTLPR allele was associated with EEV modulation of activity in the same region of VMPFC as that reported for the $A D R A 2 b$ deletion variant. Carrying the short allele influences serotonin activity and has been associated with greater amygdala sensitivity to emotional salience (Munafò et al., 2008) and altered patterns of connectivity between the amygdala and ventral PFC (Heinz et al., 2005), which in turn have been linked to biases in affective processing. Our finding suggests that, like deletion carriers, short allele carriers' subjective experience of emotional stimuli is more intensely colored by more abstract evaluations of emotional value. Overall this finding is consistent with evidence that NE and serotonin systems mutually modulate each other (Sara and Bouret, 2012). Future research can examine potentially distinct contributions of ADRA2b and 5HTTLPR to affective biasing of specific components of attention (e.g., orienting vs difficulty disengaging).

One outstanding question concerns whether the enhanced tuning to emotionally salient stimuli that we have observed in $A D R A 2 b$ deletion carriers is due to faster or more intense emotional learning. Studies in rodents suggest that, in development, when $\alpha 2 \mathrm{~b}$ receptors mature, emotional learning is strongly reduced (Sullivan and Wilson, 1994). These findings lead to the hypothesis that deletion carriers, who have reduced $\alpha 2 \mathrm{~b}$ inhibitory function, should show facilitated learning of emotional associations. Future research can specifically test this hypothesis.

In conclusion, $A D R A 2 b$ deletion carriers perceive emotional aspects of the world more vividly, an experience that is reflected by overall greater activity in key hubs of valuation networks. Our data suggest that common genetic differences influencing $\mathrm{NE}$ and serotonin activity-likely in conjunction with life experiencetune brain and behavior to what we learn to be emotionally important. Such emotionally enhanced perception may in part explain why deletion carriers are susceptible to intrusive memories following trauma (de Quervain and Papassotiropoulos, 2006).

\section{Notes}

Supplemental material for this article is available at http://mclab.psych. ubc.ca/wp-content/uploads/2015/01/ToddEtAl_2015_S u p p l e m e n t a r y . p d f. The supplemental material contains additional results ob- tained from analyses of recognition memory data obtained on-line 1 week after images were first encountered in the scanner. These analyses examine the relation between ADRA2b, image category, and recognition memory accuracy. We also present additional path models that were tested, as well as their fit indices, describing the influence of EEV on brain activity. This material has not been peer reviewed.

\section{References}

Akaike H (1974) A new look at the statistical model identification. IEEE Trans Automat Contr 19:716-723. CrossRef

Andrews-Hanna JR, Reidler JS, Sepulcre J, Poulin R, Buckner RL (2010) Functional-anatomic fractionation of the brain's default network. Neuron 65:550-562. CrossRef Medline

Astafiev SV, Snyder AZ, Shulman GL, Corbetta M (2010) Comment on "Modafinil shifts human locus coeruleus to low-tonic, high-phasic activity during functional MRI" and "Homeostatic sleep pressure and responses to sustained attention in the suprachiasmatic area." Science 328: 309, author reply 309. CrossRef Medline

Aston-Jones G, Cohen JD (2005) An integrative theory of locus coeruleusnorepinephrine function: adaptive gain and optimal performance. Annu Rev Neurosci 28:403-450. CrossRef Medline

Barbas H, Ghashghaei H, Dombrowski SM, Rempel-Clower NL (1999) Medial prefrontal cortices are unified by common connections with superior temporal cortices and distinguished by input from memory-related areas in the rhesus monkey. J Comp Neurol 410:343-367. CrossRef Medline

Barrett P (2007) Structural equation modelling: adjusting model fit. Personal Individ Differ 42:815-824. CrossRef

Brett M, Anton J-L, Valabregue R, Poiine J-B (2002) Region of interest analysis using an SPM toolbox [abstract]. In: 8th International Conference on Functional Mapping of the Human Brain. Sendai, Japan.

Byrne BM (1998) Structural equation modeling with LISREL, PRELIS and SIMPLIS: basic concepts, applications and programming. Mahwah, NJ: Lawrence Erlbaum Associates.

Canli T (2008) Toward a neurogenetic theory of neuroticism. Ann N Y Acad Sci 1129:153-174. CrossRef Medline

Canli T, Lesch KP (2007) Long story short: the serotonin transporter in emotion regulation and social cognition. Nat Neurosci 10:1103-1109. CrossRef Medline

Carmichael ST, Price JL (1996) Connectional networks within the orbital and medial prefrontal cortex of macaque monkeys. J Comp Neurol 371: 179-207. CrossRef Medline

Cavada C, Compañy T, Tejedor J, Cruz-Rizzolo RJ, Reinoso-Suárez F (2000) The anatomical connections of the macaque monkey orbitofrontal cortex. A review. Cereb Cortex 10:220-242. CrossRef Medline

Chikazoe J, Lee DH, Kriegeskorte N, Anderson AK (2014) Population coding of affect across stimuli, modalities and individuals. Nat Neurosci 17: 1114-1122. CrossRef Medline

Cornelius BL, Groothoff JW, van der Klink JJ, Brouwer S (2013) The performance of the K10, K6 and GHQ-12 to screen for present state DSM-IV disorders among disability claimants. BMC Public Health 13:128. CrossRef Medline

Cousijn H, Rijpkema M, Qin S, van Marle HJ, Franke B, Hermans EJ, van Wingen G, Fernández G (2010) Acute stress modulates genotype effects on amygdala processing in humans. Proc Natl Acad Sci U S A 107:98679872. CrossRef Medline

Croxson PL, Johansen-Berg H, Behrens TE, Robson MD, Pinsk MA, Gross CG, Richter W, Richter MC, Kastner S, Rushworth MF (2005) Quantitative investigation of connections of the prefrontal cortex in the human and macaque using probabilistic diffusion tractography. J Neurosci 25 : 8854-8866. CrossRef Medline

de Quervain DJ, Papassotiropoulos A (2006) Identification of a genetic cluster influencing memory performance and hippocampal activity in humans. Proc Natl Acad Sci U S A 103:4270-4274. CrossRef Medline

de Quervain DJ, Kolassa IT, Ertl V, Onyut PL, Neuner F, Elbert T, Papassotiropoulos A (2007) A deletion variant of the alpha2b-adrenoceptor is related to emotional memory in Europeans and Africans. Nat Neurosci 10:1137-1139. CrossRef Medline

Donner TH, Nieuwenhuis S (2013) Brain-wide gain modulation: the rich get richer. Nat Neurosci 16:989-990. CrossRef Medline

Friston KJ, Holmes AP, Poline JB, Grasby PJ, Williams SC, Frackowiak RS, Turner R (1995) Analysis of fMRI time-series revisited. Neuroimage 2:45-53. CrossRef Medline 
Gallagher M, Holland PC (1994) The amygdala complex: multiple roles in associative learning and attention. Proc Natl Acad Sci U S A 91:1177111776. CrossRef Medline

Grill-Spector K, Kushnir T, Hendler T, Edelman S, Itzchak Y, Malach R (1998) A sequence of object-processing stages revealed by fMRI in the human occipital lobe. Hum Brain Mapp 6:316-328. CrossRef Medline

Guerin SA, Robbins CA, Gilmore AW, Schacter DL (2012) Interactions between visual attention and episodic retrieval: dissociable contributions of parietal regions during gist-based false recognition. Neuron 75:1122_ 1134. CrossRef Medline

Hamann S, Canli T (2004) Individual differences in emotion processing. Curr Opin Neurobiol 14:233-238. CrossRef Medline

Hariri AR, Weinberger DR (2003) Functional neuroimaging of genetic variation in serotonergic neurotransmission. Genes Brain Behav 2:341-349. CrossRef Medline

Heinz A, Braus DF, Smolka MN, Wrase J, Puls I, Hermann D, Klein S, Grüsser SM, Flor H, Schumann G, Mann K, Büchel C (2005) Amygdalaprefrontal coupling depends on a genetic variation of the serotonin transporter. Nat Neurosci 8:20-21. CrossRef Medline

Hooper D, Coughlan J, Mullen MR (2008) Structural equation modelling: guidelines for determining model fit. Electronic J Bus Res Methods 6:53-60.

Hu LT, Bentler PM (1999) Cutoff criteria for fit indexes in covariance structure analysis: conventional criteria versus new alternatives. Struct Equation Model 6:1-55. CrossRef

Itti L, Koch C (2001) Computational modelling of visual attention. Nat Rev Neurosci 2:194-203. CrossRef Medline

Jenkinson M (2003) Fast, automated, N-dimensional phase-unwrapping algorithm. Magn Reson Med 49:193-197. CrossRef Medline

Jezzard P, Balaban RS (1995) Correction for geometric distortion in echo planar images from B0 field variations. Magn Reson Med 34:65-73. CrossRef Medline

Jones BE, Moore RY (1977) Ascending projections of the locus coeruleus in the rat. II. Autoradiographic study. Brain Res 127:25-53. CrossRef Medline

Kessler RC, Andrews G, Colpe LJ, Hiripi E, Mroczek DK, Normand SL, Walters EE, Zaslavsky AM (2002) Short screening scales to monitor population prevalences and trends in nonspecific psychological distress. Psychol Med 32:959-976. CrossRef Medline

Markovic J, Anderson AK, Todd RM (2014) Tuning to the significant: neu$\mathrm{ral}$ and genetic processes underlying affective enhancement of visual perception and memory. Behav Brain Res 259:229-241. CrossRef Medline

Montag C, Buckholtz JW, Hartmann P, Merz M, Burk C, Hennig J, Reuter M (2008) COMT genetic variation affects fear processing: psychophysiological evidence. Behav Neurosci 122:901-909. CrossRef Medline

Mulaik SA, James LR, Van Alstine J, Bennet N, Lins S, Stilwell CD (1989) Evaluation of goodness-of-fit Indices for structural equation models. Psychol Bull 105:430-445. CrossRef

Munafò MR, Brown SM, Hariri AR (2008) Serotonin transporter (5-HTTLPR) genotype and amygdala activation: a meta-analysis. Biol Psychiatry 63:852-857. CrossRef Medline

Nee DE, Brown JW, Askren MK, Berman MG, Demiralp E, Krawitz A, Jonides J (2013) A meta-analysis of executive components of working memory. Cereb Cortex 23:264-282. CrossRef Medline

Pessoa L (2010) Emotion and cognition and the amygdala: from "what is it?" to "what's to be done?." Neuropsychologia 48:3416-3429. CrossRef Medline

Pourtois G, Schettino A, Vuilleumier P (2013) Brain mechanisms for emo- tional influences on perception and attention: what is magic and what is not. Biol Psychol 92:492-512. CrossRef Medline

Price JL, Carmichael ST, Drevets WC (1996) Networks related to the orbital and medial prefrontal cortex; a substrate for emotional behavior? Prog Brain Res 107:523-536. CrossRef Medline

Rasch B, Spalek K, Buholzer S, Luechinger R, Boesiger P, Papassotiropoulos A, de Quervain DJ (2009) A genetic variation of the noradrenergic system is related to differential amygdala activation during encoding of emotional memories. Proc Natl Acad Sci U S A 106:19191-19196. CrossRef Medline

Reinhard E, Stark M, Shirley P, Ferwerda J (2002) Photographic tone reproduction for digital images. In: 29th Annual Conference on Computer Graphics and Interactive Techniques (SIGGRAPH), pp 267-276.

Rempel-Clower NL, Barbas H (1998) Topographic organization of connections between the hypothalamus and prefrontal cortex in the rhesus monkey. J Comp Neurol 398:393-419. CrossRef Medline

Roozendaal B, McEwen BS, Chattarji S (2009) Stress, memory and the amygdala. Nat Rev Neurosci 10:423-433. CrossRef Medline

Rottschy C, Langner R, Dogan I, Reetz K, Laird AR, Schulz JB, Fox PT, Eickhoff SB (2012) Modelling neural correlates of working memory: a coordinatebased meta-analysis. Neuroimage 60:830-846. CrossRef Medline

Sara SJ (2009) The locus coeruleus and noradrenergic modulation of cognition. Nat Rev Neurosci 10:211-223. CrossRef Medline

Sara SJ, Bouret S (2012) Orienting and reorienting: the locus coeruleus mediates cognition through arousal. Neuron 76:130-141. CrossRef Medline

Smolka MN, Schumann G, Wrase J, Grüsser SM, Flor H, Mann K, Braus DF, Goldman D, Büchel C, Heinz A (2005) Catechol-O-methyltransferase val158met genotype affects processing of emotional stimuli in the amygdala and prefrontal cortex. J Neurosci 25:836-842. CrossRef Medline

Sullivan RM, Wilson DA (1994) The locus coeruleus, norepinephrine, and memory in newborns. Brain Res Bull 35:467-472. CrossRef Medline

Todd RM, Talmi D, Schmitz TW, Susskind J, Anderson AK (2012) Psychophysical and neural evidence for emotion-enhanced perceptual vividness. J Neurosci 32:11201-11212. CrossRef Medline

Todd RM, Müller DJ, Lee DH, Robertson A, Eaton T, Freeman N, Palombo DJ, Levine B, Anderson AK (2013) Genes for emotion-enhanced remembering are linked to enhanced perceiving. Psychol Sci 24:2244-2253. CrossRef Medline

Todd RM, Müller DJ, Palombo DJ, Robertson A, Eaton T, Freeman N, Levine $\mathrm{B}$, Anderson AK (2014) Deletion variant in the ADRA2B gene increases coupling between emotional responses at encoding and later retrieval of emotional memories. Neurobiol Learn Mem 112:222-229. CrossRef Medline

Tzourio-Mazoyer N, Landeau B, Papathanassiou D, Crivello F, Etard O, Delcroix N, Mazoyer B, Joliot M (2002) Automated anatomical labeling of activations in SPM using a macroscopic anatomical parcellation of the MNI MRI single-subject brain. Neuroimage 15:273-289. CrossRef Medline

Walther D, Koch C (2006) Modeling attention to salient proto-objects. Neural Netw 19:1395-1407. CrossRef Medline

Wang HE, Bénar CG, Quilichini PP, Friston KJ, Jirsa VK, Bernard C (2014) A systematic framework for functional connectivity measures. Front Neurosci 8:405. CrossRef Medline

Waterhouse BD, Azizi SA, Burne RA, Woodward DJ (1990) Modulation of rat cortical area 17 neuronal responses to moving visual stimuli during norepinephrine and serotonin microiontophoresis. Brain Res 514:276292. CrossRef Medline

Yu AJ, Dayan P (2005) Uncertainty, neuromodulation, and attention. Neuron 46:681-692. CrossRef Medline 